\title{
A note about maximal almost-invariant subspaces and maximal hyperinvariant subspaces
}

\author{
Cui Chen ${ }^{1} \cdot$ Ze-Hua Zhou ${ }^{2} \cdot$ Ya Wang ${ }^{1}$
}

Published online: 27 June 2019

(c) The Author(s) 2019

\begin{abstract}
In this paper, we show that for $T \in B(\mathcal{H})$, if $\mathcal{M}$ is almost-invariant for $T$, then every maximal almost-invariant subspace of $\mathcal{M}$ is of codimension 1 in $\mathcal{M}$, where $\mathcal{H}$ is a separable, infinite-dimensional Hilbert space. We also describe the maximal hyperinvariant subspaces for normal operators with all the dimensions of eigenspaces at most 1 acting on $\mathcal{H}$. Our result is that for each hyperinvariant subspace, all its maximal hyperinvariant subspaces are also of codimension 1 in it.
\end{abstract}

Keywords Maximal almost-invariant subspaces · Maximal hyperinvariant subspaces · Codimension

Mathematics Subject Classification Primary 47A15; Secondary 47L05

\section{Introduction}

Let $\mathcal{H}$ be a separable, infinite-dimensional Hilbert space and denote by $B(\mathcal{H})$ the set of bounded linear operators acting on $\mathcal{H}$. For $T \in B(\mathcal{H})$ a subspace $\mathcal{M}$ of $\mathcal{H}$ is called invariant for $T$, or $T$-invariant, if it is closed and $T \mathcal{M} \subseteq \mathcal{M}$. The classical Invariant Subspace Problem, one of the most important problems in Operator Theory, is about the existence of non-trivial invariant subspaces for an operator $T \in B(\mathcal{H})$.

The work was supported by the National Natural Science Foundation of China (Grant No. 11771323).

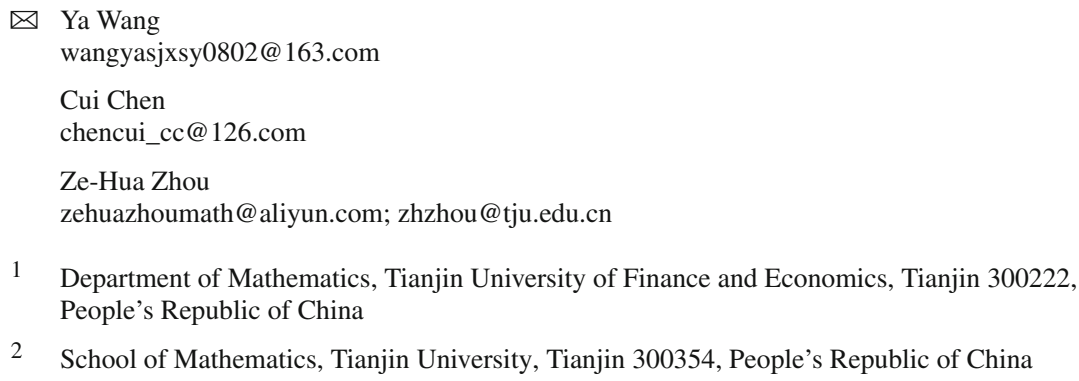

2 School of Mathematics, Tianjin University, Tianjin 300354, People's Republic of China 
For an operator $T \in B(\mathcal{H})$ and an invariant subspace $\mathcal{M}$ for $T$, a $T$-invariant subspace $\mathcal{N}$ is called a maximal invariant subspace of $\mathcal{M}$, if $\mathcal{N} \subsetneq \mathcal{M}$ and there is no $T$-invariant subspace $\mathcal{L}$ such that $\mathcal{N} \subsetneq \mathcal{L} \subsetneq \mathcal{M}$. Hedenmalm [6] obtained first the result that every maximal invariant subspace of the Bergman space is of codimension 1. For further generalizions of the Bergman space, we refer the interested readers to [1,16]. Later, Guo et al. [5] extended the result to a much more general situation. Their result is the following.

Theorem 1.1 ([5, Theorem 1.1]). Suppose $T$ is a Fredholm operator acting on a separable Hilbert space and $1-T T^{*} \in \mathcal{S}_{p}$ for some $p \geq 1$. If $\mathcal{M}$ is an invariant subspace for $T$ such that $\operatorname{dim} \mathcal{M} \Theta T \mathcal{M}<\infty$, then every maximal invariant subspace of $\mathcal{M}$ is of codimension 1 in $\mathcal{M}$.

Here $\mathcal{S}_{p}(p>0)$ denotes the set of Schatten- $p$ class operators; and for two subspaces $\mathcal{U}, \mathcal{V}$ of $\mathcal{H}, \mathcal{U} \Theta \mathcal{V}=\mathcal{U} \cap \mathcal{V}^{\perp}$, where $\mathcal{V}^{\perp}$ denotes the orthogonal complement space of $\mathcal{V}$ in $\mathcal{H}$.

Motivated by the above work, we intend to study the maximal almost-invariant subspaces and maximal hyperinvariant subspaces.

A subspace $\mathcal{M}$ of $\mathcal{H}$ is called almost-invariant for $T$ (or $T$-almost invariant) if it is closed and $T \mathcal{M} \subseteq \mathcal{M}+\mathcal{F}$ for some finite-dimensional subspace $\mathcal{F}$ of $\mathcal{H}$. This concept was first introduced in [2]. The minimal dimension of such a subspace $\mathcal{F}$ is referred to as the defect of $\mathcal{M}$ for $T$. It is obvious that every finite-dimensional or finite-codimensional subspace is almost-invariant under $T$. So we only need to consider a half-space, that is, a subspace of $\mathcal{H}$ which is infinite-dimensional and infinite-codimensional. For further information about almost-invariant subspaces, we refer the interested readers to [2,12-14].

In a similar way, we give the definition of maximal almost-invariant subspace.

Definition 1.2 For an operator $T \in B(\mathcal{H})$ and an almost-invariant subspace $\mathcal{M}$ for $T$, a Talmost invariant subspace $\mathcal{N}$ is called a maximal almost-invariant subspace of $\mathcal{M}$, if $\mathcal{N} \subsetneq \mathcal{M}$ and there is no $T$-almost invariant subspace $\mathcal{L}$ such that $\mathcal{N} \subsetneq \mathcal{L} \subsetneq \mathcal{M}$.

We will prove that, given an operator $T \in B(\mathcal{H})$, for any $T$-almost invariant half-space $\mathcal{M}$ every maximal almost-invariant subspace of $\mathcal{M}$ is of codimension 1 in $\mathcal{M}$.

A subspace $\mathcal{M}$ of $\mathcal{H}$ is called hyperinvariant for $T$, or $T$-hyperinvariant, if it is closed and $W \mathcal{M} \subseteq \mathcal{M}$ for each $W \in\{T\}^{\prime}$. Here $\{T\}^{\prime}$ denotes the commutant of $T$ given by

$$
\{T\}^{\prime}=\{W \in B(\mathcal{H}): W T=T W\} .
$$

There are many unsolved problems in the theory of invariant subspaces, hence these problems need close attention. In this paper, we first deal with hyperinvariant subspaces. For a further discussion about hyperinvariant subspaces, we recommend to the interested readers the recent papers $[4,7-11,15]$.

We also define maximal hyperinvariant subspaces analogously.

Definition 1.3 For an operator $T \in B(\mathcal{H})$ and a hyperinvariant subspace $\mathcal{M}$ for $T$, a Thyperinvariant subspace $\mathcal{N}$ is called a maximal hyperinvariant subspace of $\mathcal{M}$, if $\mathcal{N} \subsetneq \mathcal{M}$ and there is no $T$-hyperinvariant subspace $\mathcal{L}$ such that $\mathcal{N} \subsetneq \mathcal{L} \subsetneq \mathcal{M}$.

An operator $T \in B(\mathcal{H})$, is said to be normal if $T^{*} T=T T^{*}$.

Our conclusion about maximal hyperinvariant subspaces is in the setting of a Hilbert space $\mathcal{H}$. We will show that for a normal operator $T \in B(\mathcal{H})$ and a $T$-hyperinvariant subspace $\mathcal{M}$, if all the dimensions of eigenspaces of $T$ are at most 1 , then every maximal hyperinvariant subspace of $\mathcal{M}$ is of codimension 1 in $\mathcal{M}$.

Throughout the paper, for a closed subspace $\mathcal{E}$ of $\mathcal{H}, P_{\mathcal{E}}$ denotes the orthogonal projection from $\mathcal{H}$ to $\mathcal{E}$ and $\left.T\right|_{\mathcal{E}}$ is the operator $T$ restricted to $\mathcal{E}$. 


\section{Maximal almost-invariant subspaces}

In this section, we give a characterization of maximal almost-invariant subspaces. The main result can be formulated as follows.

Theorem 2.1 For $T \in B(\mathcal{H})$, if $\mathcal{M}$ is a $T$-almost invariant half-space, then every maximal almost-invariant subspace of $\mathcal{M}$ is of codimension 1 in $\mathcal{M}$.

The first step in the proof of Theorem 2.1 is the following lemma.

Lemma 2.2 Let $T \in B(\mathcal{H})$. Suppose $\mathcal{M}$ and $\mathcal{N}$ are two $T$-almost invariant half-spaces with $\mathcal{N} \subsetneq \mathcal{M}$ and $\operatorname{dim} \mathcal{M} \Theta \mathcal{N} \geq 2$. Put $S=\left.P_{M \Theta N} T\right|_{M \Theta N}$. Then $T$ has an almost invariant halfspace $\mathcal{L}$ such that $\mathcal{N} \subsetneq \mathcal{L} \subsetneq \mathcal{M}$ if and only if there exists an $S$-almost invariant subspace $\mathcal{L}_{0}$ such that $0 \subsetneq \mathcal{L}_{0} \subsetneq \mathcal{M} \Theta \mathcal{N}$.

Proof Since $\mathcal{M}, \mathcal{N}$ are both $T$-almost invariant half-spaces, we write $T \mathcal{M} \subseteq \mathcal{M}+\mathcal{F}_{1}$ and $T \mathcal{N} \subseteq \mathcal{N}+\mathcal{F}_{2}$, where $\mathcal{F}_{1}$ and $\mathcal{F}_{2}$ are finite-dimensional subspaces of $\mathcal{H}$. Now, if $\mathcal{L}$ is almostinvariant for $T$ with $\mathcal{N} \subsetneq \mathcal{L} \subsetneq \mathcal{M}$, we assume that $T \mathcal{L} \subseteq \mathcal{L}+\mathcal{F}_{3}$ for some finite-dimensional subspace $\mathcal{F}_{3}$. Put $\mathcal{L}_{0}=\mathcal{L} \Theta \mathcal{N}$; then it is clear that $0 \subsetneq \mathcal{L}_{0} \subsetneq \mathcal{M} \Theta \mathcal{N}$, and

$$
S \mathcal{L}_{0}=\left.P_{\mathcal{M} \Theta \mathcal{N}} T\right|_{\mathcal{M} \Theta \mathcal{N}}(\mathcal{L} \Theta \mathcal{N}) \subseteq\left(\mathcal{L}+\mathcal{F}_{3}\right) \Theta \mathcal{N} \subseteq \mathcal{L} \Theta \mathcal{N}+\mathcal{F}_{3} \subseteq \mathcal{L}_{0}+\mathcal{F}_{3},
$$

that is $\mathcal{L}_{0}$ is $S$-almost invariant.

Conversely, we assume that there exists a subspace $\mathcal{L}_{0}$ with $0 \subsetneq \mathcal{L}_{0} \subsetneq \mathcal{M} \Theta \mathcal{N}$ that is $S$ almost invariant by $S \mathcal{L}_{0} \subseteq \mathcal{L}_{0}+\mathcal{F}_{4}$ for some finite-dimensional subspace $\mathcal{F}_{4}$. Put $\mathcal{L}=\mathcal{L}_{0}+$ $\mathcal{N}$, then $\mathcal{L}$ is half-space and $\mathcal{N} \subsetneq \mathcal{L} \subsetneq \mathcal{M}$. Next we prove that $\mathcal{L}$ is $T$-almost invariant. Noting that $S \mathcal{L}_{0} \subseteq \mathcal{L}_{0}+\mathcal{F}_{4}$, i.e., $\left.P_{\mathcal{M} \Theta \mathcal{N}} T\right|_{\mathcal{M} \Theta \mathcal{N}} \mathcal{L}_{0} \subseteq \mathcal{L}_{0}+\mathcal{F}_{4}$, we have $P_{\mathcal{N} \perp} T \mathcal{L}_{0} \subseteq \mathcal{L}_{0}+\mathcal{F}_{4}+\mathcal{F}_{1}$. Then $T \mathcal{L}_{0}=P_{\mathcal{N}} T \mathcal{L}_{0}+P_{\mathcal{N} \perp} T \mathcal{L}_{0} \subseteq \mathcal{N}+\mathcal{L}_{0}+\mathcal{F}_{4}+\mathcal{F}_{1}$, thus

$$
T \mathcal{L}=T \mathcal{L}_{0}+T \mathcal{N} \subseteq \mathcal{L}+\mathcal{F}_{4}+\mathcal{F}_{1}+\mathcal{N}+\mathcal{F}_{2} \subseteq \mathcal{L}+\widehat{\mathcal{F}}
$$

for some finite-dimensional subspace $\widehat{\mathcal{F}}$, that is, $\mathcal{L}$ is $T$-almost invariant. So the assertion of this lemma is proved.

The following lemma, proved by Popov and Tcaciuc in [14], is quite important to get the main result of this section.

Lemma 2.3 Let $T$ be a bounded operator on an infinite-dimensional, reflexive Banach space $\mathcal{X}$. Then $\mathcal{X}$ admits an almost-invariant half-space with defect 1.

Using this lemma, we can prove the following result, which is the key idea of the proof of Theorem 2.1.

Lemma 2.4 Let $T \in B(\mathcal{H})$. Suppose $\mathcal{M}$ and $\mathcal{N}$ are two $T$-almost invariant half-spaces with $\mathcal{N} \subsetneq \mathcal{M}$ and $\operatorname{dim} \mathcal{M} \Theta \mathcal{N} \geq 2$. Then there is a $T$-almost invariant half-space $\mathcal{L}$ such that $\mathcal{N} \subsetneq \mathcal{L} \subsetneq \mathcal{M}$.

Proof Set $T \mathcal{M} \subseteq \mathcal{M}+\mathcal{F}_{1}$ and $T \mathcal{N} \subseteq \mathcal{N}+\mathcal{F}_{2}$ as in the proof of Lemma 2.2. Firstly, assuming that $\operatorname{dim} \mathcal{M} \Theta \mathcal{N}<\infty$, we can choose half-space $\mathcal{L}$ such that $\mathcal{N} \subsetneq \mathcal{L} \subsetneq \mathcal{M}$ since $\operatorname{dim} \mathcal{M} \Theta \mathcal{N} \geq 2$. Moreover, for each half-space $\mathcal{L}$ with $\mathcal{N} \subsetneq \mathcal{L} \subsetneq \mathcal{M}$, we have

$$
T \mathcal{L} \subseteq T \mathcal{M} \subseteq T(\mathcal{M} \Theta \mathcal{N})+T \mathcal{N} \subseteq T(\mathcal{M} \Theta \mathcal{N})+\mathcal{N}+\mathcal{F}_{2} \subseteq \mathcal{L}+\widetilde{\mathcal{F}},
$$

for some finite-dimensional subspace $\widetilde{\mathcal{F}}$ of $\mathcal{H}$ since $\operatorname{dim} \mathcal{M} \Theta \mathcal{N}<\infty$. 
Now we assume $\operatorname{dim} \mathcal{M} \Theta \mathcal{N}=\infty$. Consider the operator $S=\left.P_{\mathcal{M} \Theta \mathcal{N}} T\right|_{\mathcal{M} \Theta \mathcal{N}}$. Since $S=$ $\left.P_{\mathcal{M} \Theta \mathcal{N}} T\right|_{\mathcal{M} \Theta \mathcal{N}} \in B(\mathcal{M} \Theta \mathcal{N})$ and $\mathcal{M} \Theta \mathcal{N}$ is an infinite-dimensional, reflexive Banach space, by Lemma $2.3, \mathcal{M} \Theta \mathcal{N}$ admits an $S$-almost invariant half-space with defect 1 . Therefore, using Lemma 2.2, there exists a $T$-almost invariant subspace $\mathcal{L}$ such that $\mathcal{N} \subsetneq \mathcal{L} \subsetneq \mathcal{M}$. This completes the proof.

Now the characterization of maximal almost-invariant subspaces is a direct consequence of this lemma.

At the end of this section, we want to pose a question to the interested readers. In the proof, the finite-dimensional subspaces making sure that $\mathcal{N}, \mathcal{L}, \mathcal{M}$ are $T$-almost invariant may not have the same dimension or even be the same subspace. Of course, here we mean such a finite-dimensional subspace with minimal dimension to make sure $\mathcal{N}, \mathcal{L}, \mathcal{M}$ are $T$-almost invariant. Hence, it is natural to ask the following question:

Question 2.5 Is there a separable, infinite-dimensional Hilbert space $\mathcal{H}$, and an operator $T \in B(\mathcal{H})$ such that there exist three half-spaces $\mathcal{N} \subsetneq \mathcal{L} \subsetneq \mathcal{M}$ that are $T$-almost invariant subspaces with the same defect or even the same finite-dimensional subspace are T-almost invariant subspaces?

\section{Maximal hyperinvariant subspaces}

In the case when we consider the maximal hyperinvariant subspaces, we focus on the normal operators acting on a separable, infinite-dimensional Hilbert space $\mathcal{H}$. The main result relies on the following lemma, which is proved in a similar way to [5, Lemma 2.3].

Lemma 3.1 Let $T \in B(\mathcal{H}), \mathcal{M}$ and $\mathcal{N}$ be two $T$-hyperinvariant subspaces with $\mathcal{N} \subsetneq \mathcal{M}$ and $\operatorname{dim} \mathcal{M} \Theta \mathcal{N} \geq 2$. Put $S=\left.P_{\mathcal{M} \Theta \mathcal{N}} T\right|_{\mathcal{M} \Theta \mathcal{N}}$. Then if $S$ has a non-trivial hyperinvariant subspace then $T$ has a hyperinvariant subspace $\mathcal{L}$ such that $\mathcal{N} \subsetneq \mathcal{L} \subsetneq \mathcal{M}$.

Moreover, if in additon $T$ is normal, then the existence of a $T$-hyperinvariant subspace $\mathcal{L}$ with $\mathcal{N} \subsetneq \mathcal{L} \subsetneq \mathcal{M}$ implies the existence of a non-trivial hyperinvariant subspace for $S$.

Proof Suppose that $\mathcal{L}_{0}$ is a nontrivial hyperinvariant subspace for $S$. It is clear that $0 \subsetneq \mathcal{L}_{0} \subsetneq$ $\mathcal{M} \Theta \mathcal{N}$. Setting $\mathcal{L}=\mathcal{L}_{0}+\mathcal{N}$, we have $\mathcal{N} \subsetneq \mathcal{L} \subsetneq \mathcal{M}$. Next we prove that $\mathcal{L}$ is hyperinvariant for $T$. For any $W \in\{T\}^{\prime}$, we first prove $\left.P_{\mathcal{M} \Theta \mathcal{N}} W\right|_{\mathcal{M} \Theta \mathcal{N}} \in\{S\}^{\prime}$. Indeed,

$$
\begin{aligned}
\left.\left.P_{\mathcal{M} \Theta \mathcal{N}} W\right|_{\mathcal{M} \Theta \mathcal{N}} P_{\mathcal{M} \Theta \mathcal{N}} T\right|_{\mathcal{M} \Theta \mathcal{N}} & =P_{\mathcal{M} \Theta \mathcal{N}} W P_{\mathcal{M} \Theta \mathcal{N}} T P_{\mathcal{M} \Theta \mathcal{N}} \\
& =P_{\mathcal{M} \Theta \mathcal{N}} W\left(P_{\mathcal{M}}-P_{\mathcal{N}}\right) T P_{\mathcal{M} \Theta \mathcal{N}} \\
& =P_{\mathcal{M} \Theta \mathcal{N}} W P_{\mathcal{M}} T P_{\mathcal{M} \Theta \mathcal{N}}-P_{\mathcal{M} \Theta \mathcal{N}} W P_{\mathcal{N}} T P_{\mathcal{M} \Theta \mathcal{N}} \\
& =P_{\mathcal{M} \Theta \mathcal{N}} W T P_{\mathcal{M} \Theta \mathcal{N}}
\end{aligned}
$$

here we used $P_{\mathcal{M} \Theta \mathcal{N}} W P_{\mathcal{N}} T P_{\mathcal{M} \Theta \mathcal{N}}=0$ since $\mathcal{N}$ is hyperinvariant for $T$ and $W \in\{T\}^{\prime}$.

In a similar way, we can obtain

$$
\begin{aligned}
\left.\left.P_{\mathcal{M} \Theta \mathcal{N}} T\right|_{\mathcal{M} \Theta \mathcal{N}} P_{\mathcal{M} \Theta \mathcal{N}} W\right|_{\mathcal{M} \Theta \mathcal{N}} & =P_{\mathcal{M} \Theta \mathcal{N}} T P_{\mathcal{M} \Theta \mathcal{N}} W P_{\mathcal{M} \Theta \mathcal{N}} \\
& =P_{\mathcal{M} \Theta \mathcal{N}} T\left(P_{\mathcal{M}}-P_{\mathcal{N}}\right) W P_{\mathcal{M} \Theta \mathcal{N}} \\
& =P_{\mathcal{M} \Theta \mathcal{N}} T P_{\mathcal{M}} W P_{\mathcal{M} \Theta \mathcal{N}}-P_{\mathcal{M} \Theta \mathcal{N}} T P_{\mathcal{N}} W P_{\mathcal{M} \Theta \mathcal{N}} \\
& =P_{\mathcal{M} \Theta \mathcal{N}} T W P_{\mathcal{M} \Theta \mathcal{N}}
\end{aligned}
$$


Since $W T=T W$, thus

$$
\left.\left.P_{\mathcal{M} \Theta \mathcal{N}} W\right|_{\mathcal{M} \Theta \mathcal{N}} P_{\mathcal{M} \Theta \mathcal{N}} T\right|_{\mathcal{M} \Theta \mathcal{N}}=\left.\left.P_{\mathcal{M} \Theta \mathcal{N}} T\right|_{\mathcal{M} \Theta \mathcal{N}} P_{\mathcal{M} \Theta \mathcal{N}} W\right|_{\mathcal{M} \Theta \mathcal{N}}
$$

that is $\left.P_{\mathcal{M} \Theta \mathcal{N}} W\right|_{\mathcal{M} \Theta \mathcal{N}} \in\{S\}^{\prime}$.

Since $\mathcal{L}_{0}$ is a hyperinvariant subspace for $S$, then $\left.P_{\mathcal{M} \Theta \mathcal{N}} W\right|_{\mathcal{M} \Theta \mathcal{N}} \mathcal{L}_{0} \subseteq \mathcal{L}_{0}$, hence we have $P_{\mathcal{N}^{\perp}} W \mathcal{L}_{0} \subseteq \mathcal{L}_{0}$. Then it is easy to see that $W \mathcal{L}_{0}=P_{\mathcal{N}} W \mathcal{L}_{0}+P_{\mathcal{N}^{\perp}} W \mathcal{L}_{0} \subseteq \mathcal{N}+\mathcal{L}_{0}=\mathcal{L}$. Therefore, we conclude that

$$
W \mathcal{L}=W \mathcal{L}_{0}+W \mathcal{N} \subseteq \mathcal{L} .
$$

That is, $\mathcal{L}$ is hyperinvariant for $T$ by the arbitrariness of $W \in\{T\}^{\prime}$, which proves the first assertion of this lemma.

Conversely, note that $T$ is normal, i.e., $T^{*} \in\{T\}^{\prime}$. Therefore, $\mathcal{M}, \mathcal{N}$ are both reducing subspaces of $T$, so is $\mathcal{M} \Theta \mathcal{N}$. Thus we conclude that $S=\left.T\right|_{\mathcal{M} \Theta \mathcal{N}}$. Then the operator $T$ has the corresponding decomposition

$$
T=S \oplus T_{1},
$$

where $T_{1}$ is the restriction of $T$ on $(\mathcal{M} \Theta \mathcal{N})^{\perp}$. Given an operator $W_{0} \in \mathcal{L}(\mathcal{M} \Theta \mathcal{N})$, if $W_{0} S=S W_{0}$, set $W=W_{0} \oplus I_{(\mathcal{M} \Theta \mathcal{N})^{\perp}}$, it follows that $W \in\{T\}^{\prime}$. Thus $W \mathcal{L} \subseteq \mathcal{L}$. It is easy to prove that $W_{0} \mathcal{L}_{0} \subseteq \mathcal{L}_{0}$, if $\mathcal{L}_{0}=\mathcal{L} \Theta \mathcal{N}$. So the second assertion of the lemma is obtained.

Lemma 3.2 Let $T \in B(\mathcal{H})$ be a normal operator, and $\mathcal{M}$ and $\mathcal{N}$ two $T$-hyperinvariant subspaces with $\mathcal{N} \subsetneq \mathcal{M}$ and $\operatorname{dim} \mathcal{M} \Theta \mathcal{N} \geq 2$. Then $S=\left.P_{\mathcal{M} \Theta \mathcal{N}} T\right|_{\mathcal{M} \Theta \mathcal{N}}$ is also normal.

Proof Since $\mathcal{M}, \mathcal{N}$ are both $T$-hyperinvariant, and $T^{*} \in\{T\}^{\prime}$, then $\mathcal{M}, \mathcal{N}$ are both reducing subspaces of $T$, so is $\mathcal{M} \Theta \mathcal{N}$. Hence $S=\left.T\right|_{\mathcal{M} \Theta \mathcal{N}}$. Next, we will show that $\left.T\right|_{\mathcal{M} \Theta \mathcal{N}} ^{*}=$ $\left.T^{*}\right|_{\mathcal{M} \Theta \mathcal{N}} \cdot$ In fact,

$$
\left\langle\left. T\right|_{\mathcal{M} \Theta \mathcal{N}} ^{*} x, y\right\rangle=\left\langle x,\left.T\right|_{\mathcal{M} \Theta \mathcal{N}} y\right\rangle=\langle x, T y\rangle=\left\langle T^{*} x, y\right\rangle=\left\langle\left. T^{*}\right|_{\mathcal{M} \Theta \mathcal{N}} x, y\right\rangle
$$

for $x, y \in \mathcal{M} \Theta \mathcal{N}$. Then the result follows from the normality of $T$.

The next result can be found in [3].

Lemma 3.3 A normal operator that is not a multiple of the identity has a non-trivial hyperinvariant subspace.

Using the previous lemmas, we are now ready to give the required generalization about maximal hyperinvariant subspaces.

Theorem 3.4 Let $T \in B(\mathcal{H})$ be a normal operator, and $\mathcal{M}$ be a $T$-hyperinvariant subspace. If all the dimensions of eigenspaces of $T$ are at most 1 , then every maximal hyperinvariant subspace of $\mathcal{M}$ is of codimension 1 in $\mathcal{M}$.

Proof Note that the condition that all the dimensions of eigenspaces of $T$ are at most 1 guarantees that $\left.P_{\mathcal{M} \Theta \mathcal{N}} T\right|_{\mathcal{M} \Theta \mathcal{N}}$ is not a multiple of the identity for each $T$-hyperinvariant subspace $\mathcal{N}$ with $\mathcal{N} \subsetneq \mathcal{M}$ and $\operatorname{dim} \mathcal{M} \Theta \mathcal{N} \geq 2$. Then the assertion comes easily from the lemmas above. 
Open Access This article is distributed under the terms of the Creative Commons Attribution 4.0 International License (http://creativecommons.org/licenses/by/4.0/), which permits unrestricted use, distribution, and reproduction in any medium, provided you give appropriate credit to the original author(s) and the source, provide a link to the Creative Commons license, and indicate if changes were made.

\section{References}

1. A. Atzmon, Maximal, minimal, and primary invariant subspaces. J. Funct. Anal. 185, 155-213 (2001)

2. G. Androulakis, A.I. Popov, A. Tcaciuc, V.G. Troitsky, Almost invariant half-spaces of operators on Banach spaces. Integer. Equat. Oper. Th. 65(4), 473-484 (2009)

3. I. Chalfnbar, J.R. Partington, Modern Approaches to the Invariant-Subspace Problem, Cambridge Tracts in Mathematics, 188 (Cambridge University Press, Cambridge, 2011)

4. C. Folas, I.B. Jung, E. Ko, C. Pearcy, Hyperinvariant subspaces for some subnormal operators. T. Am. Math. Soc. 359(6), 2899-2913 (2007)

5. K.Y. Guo, W. He, S.Z. Hou, Maximal invariant subspaces for a class of operators. Ark. Math. 48, 323-333 (2010)

6. H. Hedenmalm, Maximal invariant subspaces in the Bergman space. Ark. Math. 36, 97-101 (1998)

7. S. Hamid, C. Onica, C. Pearcy, On the hyperinvariant subspace problem. II. Indiana U. Math. J. 54(3), 743-754 (2005)

8. H.J. Kim, Hyperinvariant subspace problem for quasinipotent operators. Integer. Equat. Oper. Th. 61, 103-120 (2008)

9. H.J. Kim, Hyperinvariant subspaces for quasinipotent operators on Hilbert spaces. J. Math. Anal. Appl. 350, 262-270 (2009)

10. H.J. Kim, Hyperinvariant subspaces for operators having a compact part. J. Math. Anal. Appl. 386, 110-114 (2012)

11. M.X. Liu, On hyperinvariant subspaces of contraction operators on a Banach space whose spectrum contains the unit circle. Acta Math. Sin. Eng. Ser. 24(9), 1471-1474 (2008)

12. L. Marcoux, A.I. Popov, H. Radjavi, On almost-invariant subspaces and approximate commutation. J. Funct. Anal. 264, 1088-1111 (2013)

13. A.I. Popov, Almost invariant half-spaces of algebras of operators. Integer. Equat. Oper. Th. 67, 247-256 (2010)

14. A.I. Popov, A. Tcaciuc, Every operator has almost-invariant subspaces. J. Funct. Anal. 265, 257-265 (2013)

15. L.Y. Shi, Y.J. Wu, Y.Q. Ji, Invariant and hyperinvariant subspaces for amenable operators. J. Operat. Theor. 69(1), 87-100 (2013)

16. T. Trent, Maximal invariant subspaces for $A_{\alpha}^{2}(D)$. Proc. Am. Math. Soc. 132, 2429-2432 (2004)

Publisher's Note Springer Nature remains neutral with regard to jurisdictional claims in published maps and institutional affiliations. 